CERN-NUFACT-Note 39

August 16, 2000

\title{
Muon Capture and Acceleration without Phase Rotation and Cooling
}

\author{
K. Hanke
}

\section{Introduction}

Phase rotation and cooling are major technical challenges for neutrino factories and muon colliders. A simplified scheme was proposed which does not include these two components [1]. The basic idea of this scheme is to capture the muons at $p_{z} \approx 300 \pm 150 \mathrm{MeV} / \mathrm{c}$ in three buckets at $35.2 \mathrm{MHz}$ (see Fig. 1), then adiabatically ramp the phase from $-90^{\circ}$ (bunching) to $0^{\circ}$ (crest) and accelerate up to $1 \mathrm{GeV}$. At $1 \mathrm{GeV}$, the frequency is changed to $105.6 \mathrm{MHz}$. Above $2 \mathrm{GeV}$, the LEP II super-conducting cavities at $352 \mathrm{MHz}$ can be used.

In the original proposal, the muons are captured by $35.2 \mathrm{MHz}$ cavities with a gradient of $2.5 \mathrm{MV} / \mathrm{m}$. The cavity length is $1 \mathrm{~m}$. Two cavities are followed by a $2 \mathrm{~m}$ long solenoid which performs transverse focusing. An unit structure has therefore a length of $4 \mathrm{~m}$ with a filling factor of $50 \%$, thus providing an average gradient of $1.25 \mathrm{MV} / \mathrm{m}$. In the simulations this turned out to be a critical point, since a separation of acceleration and focusing involves a rather complicated transverse matching from cell to cell. It was therefore decided to do the simulation with a solenoid field which is superimposed on the cavities. Such structures appear feasible and are proposed e.g. in [2]. The result of the simulations is an upper limit for the original scenario, since a scheme with separated acceleration and focusing will be longer and more complex and the muon yield will be at best the same.

This paper presents simulations of the beam dynamics for the first part of the scheme using the simulation code PATH.

\section{Capture and Bunching}

The simulations start from a distribution of $\pi^{+}$and $\pi^{-}$generated by $2.2 \mathrm{GeV}$ protons on a $26 \mathrm{~mm}$ mercury target [3]. A drift channel of $150 \mathrm{~m}$ allows all pions to decay to muons and to build up the desired correlation in longitudinal phase space. Transverse focusing is provided by a $18 \mathrm{kG}$ solenoid field. The decay channel does not contain any rf system for phase rotation. The muon distribution in longitudinal phase space at the exit of the decay channel is shown in Fig. 2.

Only a limited number of bunches can be successfully captured as the the bunching efficiency depends strongly on the initial beam energy. That is, for a given 
distribution with a large energy spread as the one shown in Fig. 2, bunches at low kinetic energy will over-rotate while those at high energy are not yet sufficiently bunched. Furthermore, it is difficult to accelerate three bunches simultaneously if their initial kinetic energy differs by a large amount. Therefore a cut is carried out at the exit of the drift section, such that a kinetic energy range between 50 and $200 \mathrm{MeV}$ is selected. This ensures that particles at very high and very low energy are not considered. Within these limits rf capture can be performed such that the obtained bunches are roughly in the same energy range. Figure 3 shows the longitudinal phase space at the exit of the capture section.

\section{Change of Synchronous Phase}

The capture section is followed by a section where the synchronous phase of the cavities is adiabatically changed from $-90^{\circ}$ (no acceleration) to $0^{\circ}$ (maximum acceleration). First, another cut in phase is performed in to select three bunches of approximately the same kinetic energy. The phase-change section consists of a total of 10 unit structures the phase of which is changed in steps of $10^{\circ}$ per cell. Transverse focusing is again ensured by a superimposed solenoid field. The distribution at the exit of this section is shown in Fig. 4.

\section{Muon Yield in Recirculator Acceptance}

At the exit of the phase change, the muon yield is calculated in order to verify at this stage if the proposed capture scheme is competitive to other schemes as e.g. [2]. The transverse phase space is shown in Fig. 5. The solid line shows the recirculator acceptance of $1.5 \pi \mathrm{cm}$ rad. The number of muons found within the acceptance corresponds to a muon yield per pion of $0.4 \%$. This yield appears presently not competitive to other schemes.

\section{Acknowledgements}

The author would like to thank D. Küchler, A. Lombardi and R. Scrivens for helpful discussions.

\section{References}

[1] K. Bongardt, private communication.

[2] A. Lombardi, A 40-80 MHz System for Phase Rotation and Cooling, CERNNUFACT-Note 20 (2000). 
[3] N. Vassilopoulos, target file sol_2gev_26mm_4mm_hg.dat.

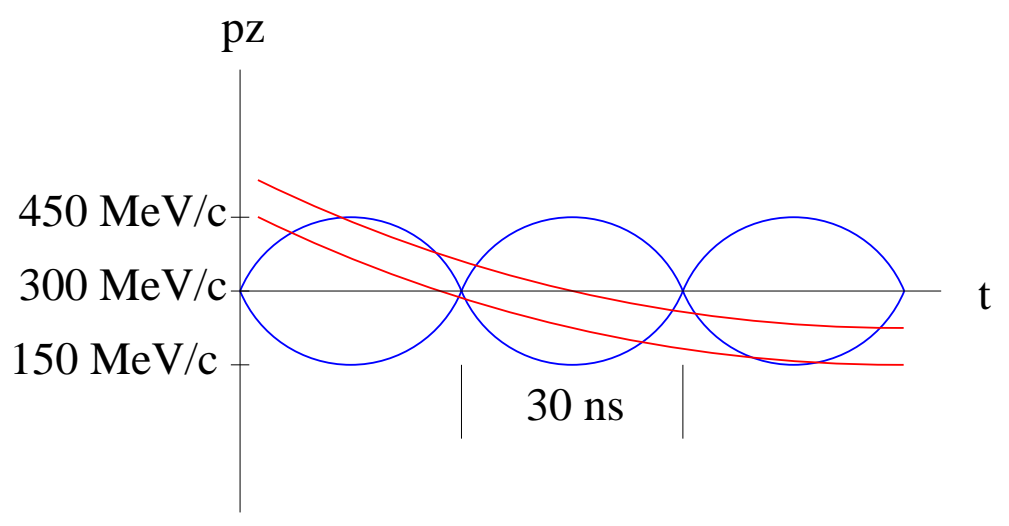

Figure 1: Schematic muon capture around $p_{z}=300 \mathrm{MeV} / \mathrm{c}$ in three buckets of 30 ns. 


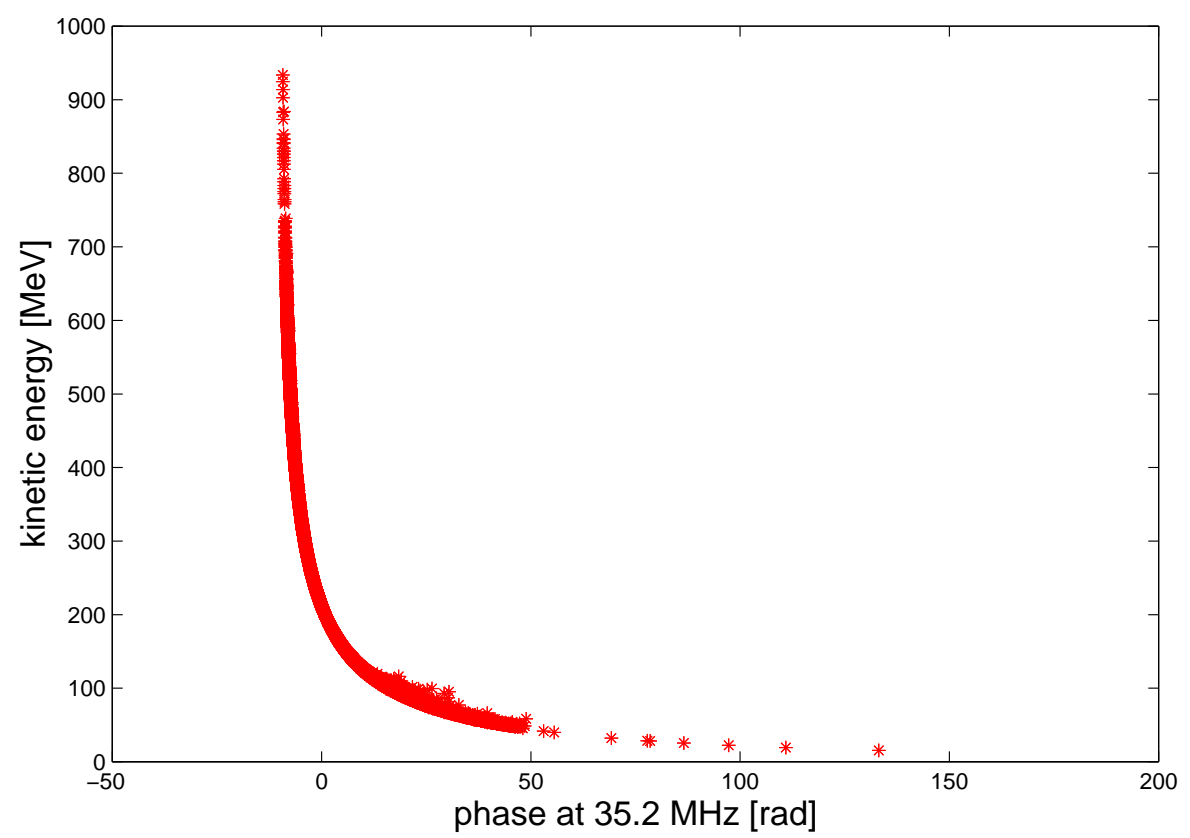

Figure 2: Longitudinal phase space distribution of muons at exit of $150 \mathrm{~m}$ solenoid decay channel.

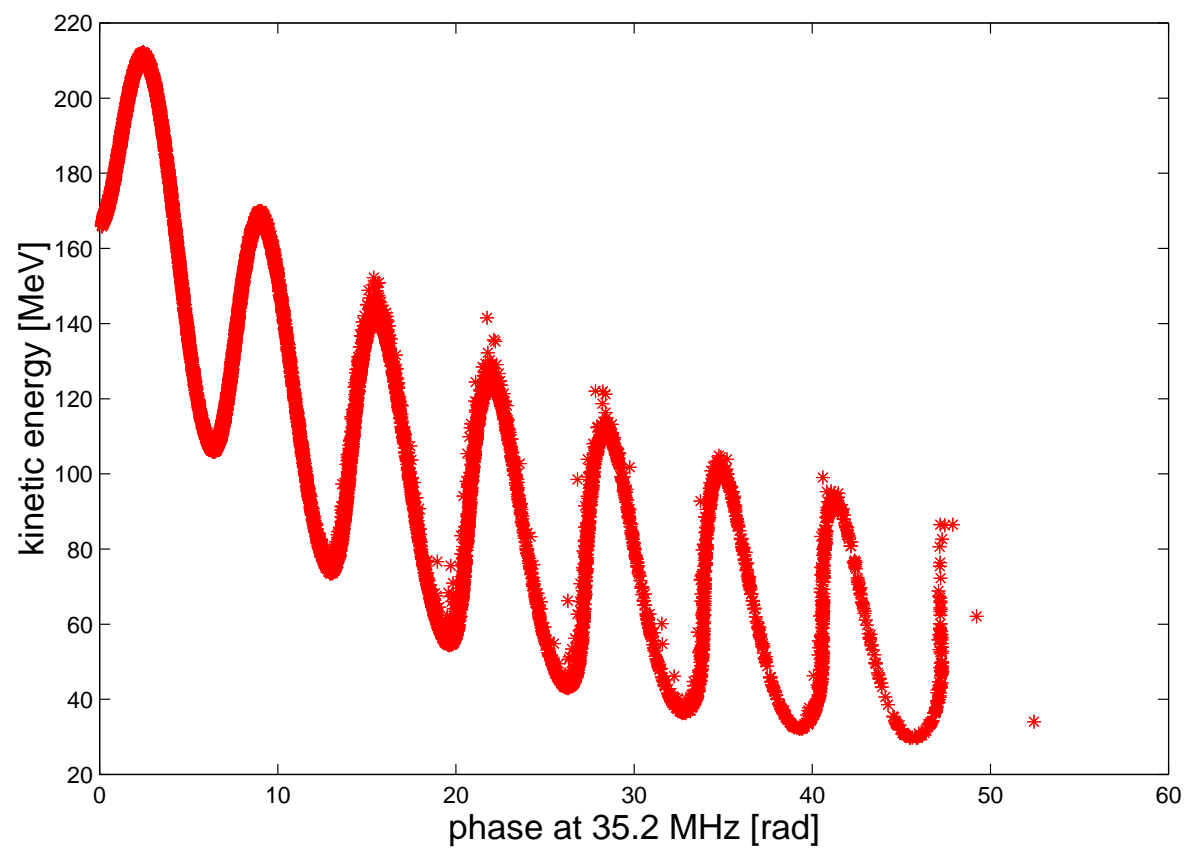

Figure 3: Longitudinal phase space distribution of muons at exit of capture section. 


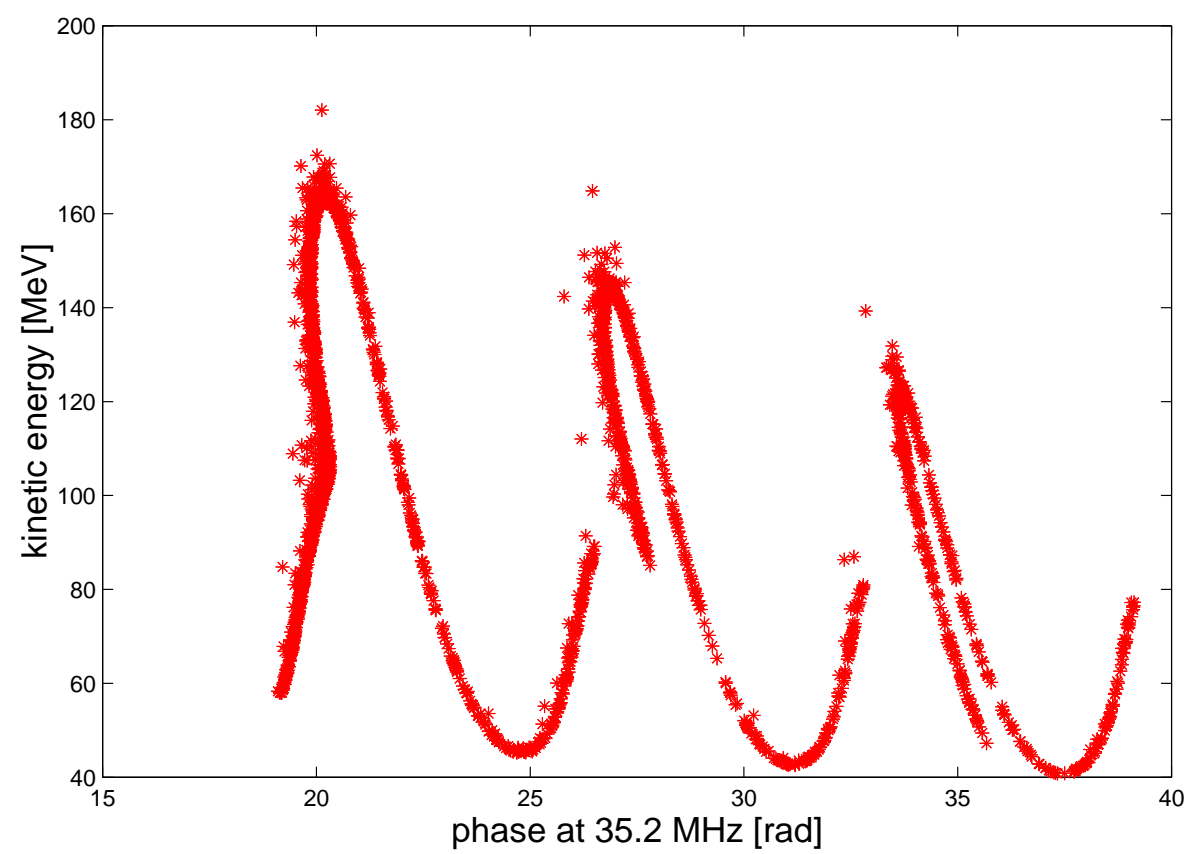

Figure 4: Longitudinal phase space distribution of muons at exit of phase change section.

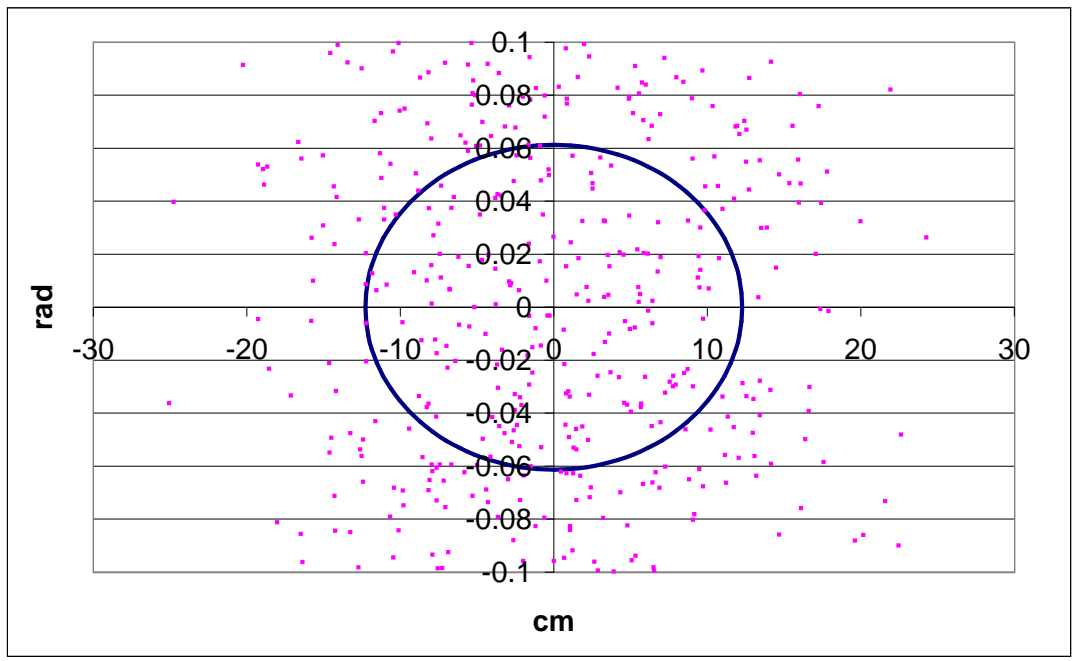

Figure 5: Muons within transverse recirculator acceptance at exit of adiabatic phase change. 